\title{
Characterisation of glass ceramic wasteforms using quantitative image analysis of electron micrographs
}

\author{
L. Harnett ${ }^{1}$ (D) M. Stennett ${ }^{1} \cdot$ E. Maddrell ${ }^{2} \cdot$ N. Hyatt $^{1}$
}

Received: 3 December 2021 / Accepted: 31 January 2022 / Published online: 9 February 2022

(c) The Author(s) 2022

\begin{abstract}
Multi-phase material systems make up a significant proportion of the currently proposed and researched wasteforms for sequestration of heterogeneous nuclear material feeds. Quantification of the components for such multi-phase assemblages is typically performed using diffraction-based Rietveld methods, many of which necessitate long measurement times of several hours. Furthermore, careful additions of an internal standard are typically required, to facilitate inclusion of amorphous phases in the quantification. The application of an image analysis method has been investigated, using the z-contrast greyscale of back-scattered electron micrographs to determine the relative quantities of component phases in a suite of monolithic phosphate glass ceramic wasteforms. This work demonstrates an alternate methodology for accelerated quantification which could be applied to other heterogeneous wasteforms and multi-phase materials.
\end{abstract}

\section{Introduction}

Quantitative phase analysis is key to the development of glass ceramic nuclear wasteforms. In addition to presenting us with relative phase ratios, it can allow us to infer properties of the material as a sum of its constituent crystalline and glassy phases. Some of the most regularly implemented phase quantification techniques have centred around X-ray diffraction-based Rietveld and pattern refinement methods. While these can provide useful additional information on material structure and phase assemblage, there are some inherent limitations such as the necessity to use an internal standard to quantify amorphous phases. Moreover, many refinement techniques require a starting model and structural understanding of the material [1,2]. Micrographic image analysis is not an uncommon technique to research areas such as cementitious materials, as it can be particularly well utilised to measure pore sizes efficiently and determine aggregate size distribution [3]. Quantitative phase analysis by electron micrograph thresholding across contrast regions

\footnotetext{
$\triangle$ L. Harnett

Lcharnett1@ sheffield.ac.uk

1 Immobilisation Science Laboratory, Department of Materials Science and Engineering, University of Sheffield, Sheffield S1 3JD, UK

2 National Nuclear Laboratory, Workington CA20 1PG, Cumbria, UK
}

has been observed in the literature, though in this example, the technique was facilitated by proprietary-hard-coded microscope software with limited flexibility and user parametrisation [4]. This work aims to provide an open-source, fast and lightweight image analysis tool to complement common characterisation techniques for glass ceramics.

\section{Materials and methods}

The prototypical glass ceramic wasteform material used in this study was from a suite of sodium-iron-phosphate compositions, with additions of alumina and silica to melts and loading of cerium oxide as a fluorite surrogate for spent fuel. The composition used for initial tests consisted of component oxides and ammonium dihydrogen phosphate, loaded with $40 \mathrm{wt} \% \mathrm{CeO}_{2}$ and melted at $1250{ }^{\circ} \mathrm{C}$ before casting and annealing for $4 \mathrm{~h}$ at $550{ }^{\circ} \mathrm{C}$, henceforth, identified as IPSAS $40 \mathrm{wt} \% \mathrm{CeO}_{2}, 1250^{\circ} \mathrm{C}$. Glass ceramic monolithic samples were sectioned, cold resin mounted and polished to a $1 \mu \mathrm{m}$ mirror finish and carbon coated to reduce surface charge. BSEs (Back scattered electron micrographs) were collected using a Hitachi TM3030 instrument, with a tungsten filament and an accelerating voltage of $15 \mathrm{kV}$. Concurrent analysis by pXRD (powder X-ray Diffraction) and EDX (Energy Dispersive X-ray) allowed for confirmation of phase assemblage [5]. Image acquisition parameters were set to medium brightness and high contrast, with fine adjustments 


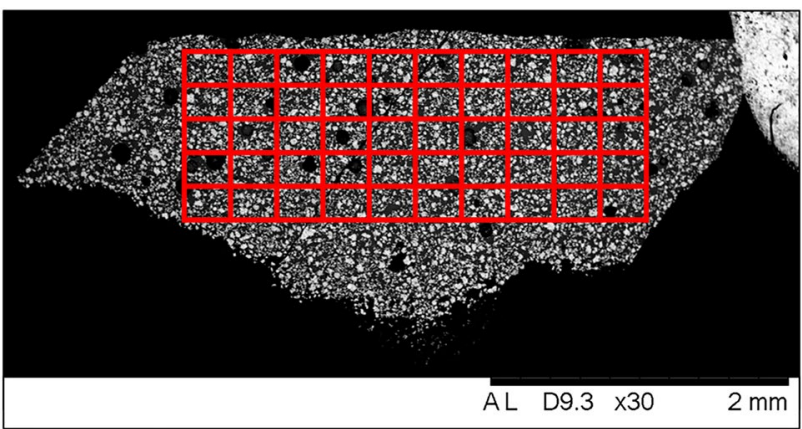

Fig. 1 Broad-overview BSE of IPSAS $40 \mathrm{wt} \% \mathrm{CeO}_{2}, 1250{ }^{\circ} \mathrm{C}$, with coordinate grid overlay

made, per sample, to maximise the contrast in grey values between the individual phases. The phase proportions can be delineated, as delimited by regions with different grey values, or BSE contrast, a factor influenced by the atomic number of the species in each phase. The free, open-source image processing software ImageJ, was used as a base platform in the Fiji package [6]. The package utilises JavaScript-based functions and macros to assist with automation of various post-processing tasks. This work concerns a script of approximately $\sim 350$ lines of code which will facilitate a quantitative-phase analysis methodology. An initial routine uses sample surface BSEs collected at a range of magnifications, measuring particle size to pixel count ratios and smallest-feature statistics. The routine is followed by selection of an optimum magnification, such that small crystallites $(>5 \mu \mathrm{m})$ are properly resolved. A low-magnification broad micrograph of the material surface was collected, allowing overlay of a coordinate grid, a pseudo-random number generator was then used to select cells of the grid to collect sample micrographs at the desired magnification, Fig. 1.

During following stages of the code progression, imagescale calibration was unnecessary, as phase quantities were determined by ratio of pixel counts. The script analyses a histogram of grey values represented in the sample BSE, then calculating the points at which minima of intensity can be found. The peak intensities between such points were then used to represent the discrete phases, the areas for which were determined by thresholding between pairs of minima and counting the number of pixels remaining, Fig. 2a. Ratios of these areas were then used to infer relative phase quantities. Following the processing of a first BSE, the remaining image set from a sample can be quickly processed to strengthen the measurement statistics. The user is given option to manually exclude mis-identified phases such as mounting material or pores Fig. $2 \mathrm{~b}$ and can also generate a representative figure to accompany phase quantity statistics for publication. At the time of writing, the script can delineate $2-6$ individual phases.

\section{Results}

Following the measurement of an initial sample BSE, the remaining image set is loaded by the script as a stack, so results are supplied as an indirect volume percentage, as the direct measurements made to individual BSEs result in area percentage values. The test sample, IPSAS $40 \mathrm{wt} \%$ $\mathrm{CeO}_{2}, 1250{ }^{\circ} \mathrm{C}$, was measured to collect statistics over a range of 10 BSEs, with the script identifying with $99.9 \%$ confidence: $61.9 \pm 2.3$ vol\% glass, $34.9 \pm 2.0$ vol $\% \mathrm{CePO}_{4}$ and $3.2 \pm 0.5$ vol\% $\mathrm{CeO}_{2}$ (darkest to lightest contrast, respectively), Fig. 3a. Another test sample, with a base
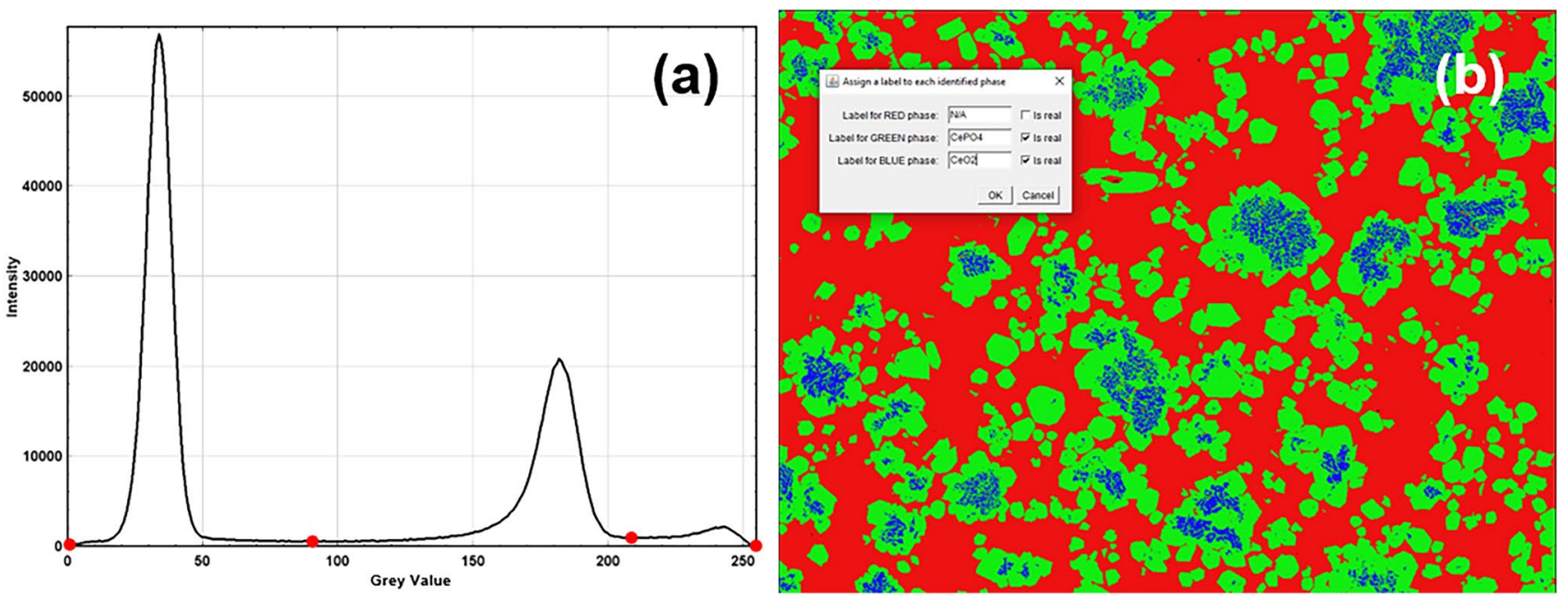

Fig. 2 Grey value histogram for IPSAS $40 \mathrm{wt} \% \mathrm{CeO}_{2}, 1250{ }^{\circ} \mathrm{C}(\mathbf{a})$, phase labelling and exclusion (b) 


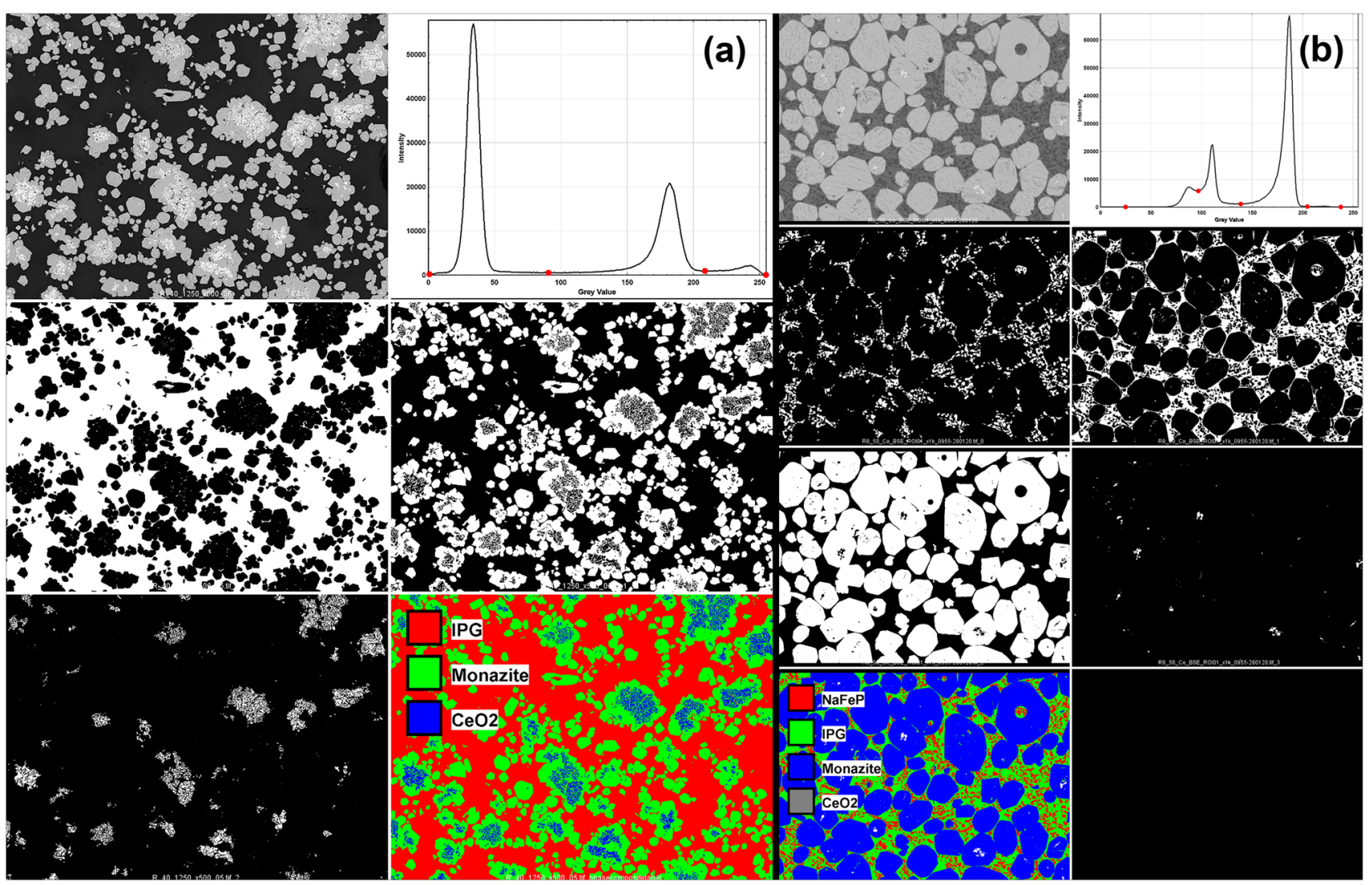

Fig. 3 Representative measurement summaries for 3-phase IPSAS $40 \mathrm{wt} \% \mathrm{CeO}_{2}, 1250{ }^{\circ} \mathrm{C}(\mathbf{a})$ and 4 -phase $\mathrm{IPSAS} 50 \mathrm{wt} \% \mathrm{CeO}_{2}, 1450{ }^{\circ} \mathrm{C}(\mathbf{b})$

sodium-iron-phosphate glass composition with $50 \mathrm{wt} \%$ $\mathrm{CeO}_{2}$ melted at $1450{ }^{\circ} \mathrm{C}$, was also measured using the same method over $10 \mathrm{BSEs}$, with $99.9 \%$ confidence: $22.0 \pm 1.7$ vol\% glass, $8.7 \pm 0.4$ vol\% crystalline sodium-iron-phosphate, $68.5 \pm 2.9$ vol $\% \mathrm{CePO}_{4}, 0.8 \pm 0.2$ vol $\% \mathrm{CeO}_{2}$ (darkest to lightest contrast, respectively), Fig. 3b. Each image was collected in about $30 \mathrm{~s}$, with another 15 for adjustment and stage movement. Image sets of stack size 10 were measured by the script in approximately $30 \mathrm{~s}$ (as verified by a counting function), on a moderately equipped workstation built in 2018 .

\section{Discussion}

The results presented here, demonstrate how the script can be used to analyse micrographs of samples with varying phase assemblages and with different phase morphologies. Some preliminary tests with a SYNROC-F type multi-phase ceramic [7] and a barium phosphate multi-phase system [8] have indicated that adjustable sensitivity will be a necessary feature to modify the minima-detecting algorithm, as some ceramic phases have surfaces with very well-defined contrast, such that the histogram of grey values presents with sharp peaks and phases with very similar contrast, such that overlap can occur. Implementation of sensitivity adjustment is underway to facilitate measurement of such phase assemblages. The technique does, however, have an inherent limitation with difficulty processing images containing phases with similar BSE grey contrast, as such, it would be unable to distinguish between two phases with exactly the same mean atomic number. A high degree of precision was achieved with the sodium-iron-phosphate glass ceramic materials, with error comparable to Rietveld-type analyses [2]; further improvements have been shown with larger samples of 20-25 BSEs. The key concluding advantage over more traditional methods often requiring several hours per sample is the data collection and processing time of imagebased QPA, represented as 8 min for a 10 BSE set, up to $20 \mathrm{~min}$ for a 25 image set. The open nature of this platform will allow for ease of additional control, modification and integration with microscopes featuring motorised or computer-controlled stages. Other heterogeneous multi-phase materials may be measured using this method, such as those studied by researchers in the fields of cultural heritage and archaeology. Such a technique may be applied to images acquired through other micro-analysis techniques such as optical and Raman spectroscopy; however, due care will be 
required to ensure defined contrast of both amorphous and crystalline phases, with minimum resolvable feature size constrained to the diffraction limit with light-based analyses. Further planned work includes measurement of samples of a zirconolite glass ceramic composition, use of a rapid X-ray diffraction-based quantification method to verify the performance and accuracy of the image method [9], improvements to portability of the analysis package-including greater compatibility through Linux and macOS platforms with their respective filesystems.

Acknowledgments This research utilised the HADES/MIDAS facility at the University of Sheffield established with financial support from EPSRC and BEIS, under Grant EP/T011424/1. The author would like to send their immeasurable gratitude to The EPSRC and Nuclear Decommissioning Authority for the award of a $\mathrm{PhD}$ studentship and appreciations to Professor Neil Hyatt and Dr Martin Stennett for their excellent support and supervision, particularly during the global pandemic.

Data availability The datasets generated during the current study are not publicly available due to active continued work and refinement of the methodology at the time of writing, subject to further publication but are available from the corresponding author on reasonable request.

\section{Declarations}

Conflict of interest No potential conflict of interest was reported by the authors.
Open Access This article is licensed under a Creative Commons Attribution 4.0 International License, which permits use, sharing, adaptation, distribution and reproduction in any medium or format, as long as you give appropriate credit to the original author(s) and the source, provide a link to the Creative Commons licence, and indicate if changes were made. The images or other third party material in this article are included in the article's Creative Commons licence, unless indicated otherwise in a credit line to the material. If material is not included in the article's Creative Commons licence and your intended use is not permitted by statutory regulation or exceeds the permitted use, you will need to obtain permission directly from the copyright holder. To view a copy of this licence, visit http://creativecommons.org/licenses/by/4.0/.

\section{References}

1. X. Orlhac et al., J. Appl. Crystallogr. 34, 114-118 (2001)

2. S. Kemethmüller et al., J. Am. Ceram. Soc. 89, 2632-2637 (2006)

3. M.A. Saghiri et al., Eur. Endod. J. 5, 128-133 (2020)

4. J. Crum et al., J. Nucl. Mater. 444, 481-492 (2014)

5. N.C. Hyatt et al., IOP Conf. Ser. Mater. Sci. Eng. 818, 012022 (2020)

6. J. Schindelin et al., Nat. Methods 9, 676-682 (2012)

7. L. Harnett et al., IOP Conf. Ser. Mater. Sci. Eng. 818, 012012 (2020)

8. K. Popa et al., J. Solid State Chem. 180, 2346-2355 (2007)

9. H.P. Klug and L.E. Alexander, X-Ray Diffraction Procedures: For Polycrystalline and Amorphous Materials, 2nd ed. (Wiley, New York, 1974) pp. 531-565 